\title{
Characterizing Instrumental Effects on Polarization at a Nasmyth focus using $\mathrm{NaCo}$
}

\author{
Jozua de Boer ${ }^{1, a, b}$, Julien H. Girard ${ }^{a}$, Dimitri Mawet ${ }^{a}$, Frans Snik ${ }^{b}$, Christoph U. Keller ${ }^{b}$ and \\ Julien Milli ${ }^{a, c}$ \\ ${ }^{a}$ European Southern Observatory, Casilla 19001, Santiago, Chile; \\ ${ }^{b}$ Leiden Observatory, Leiden University, P.O. Box 9513, 2300 RA Leiden, The Netherlands; \\ ${ }^{c}$ IPAG, University Joseph Fourier, CNRS, BP 53, 38041 Grenoble, France.
}

\begin{abstract}
We propose a new calibration scheme to determine the instrumental polarization (IP) and crosstalk induced by either the telescope or an instrument at Nasmyth focus. We measure the polarized blue sky at zenith with VLT/UT4/NaCo for different NaCo derotator and telescope azimuth angles. Taking multiple measurements after rotating both the instrument and the telescope with angles of $90^{\circ}$ allows use to determine the IP and most crosstalk components separately for the telescope and the instrument. This separation of the Mueller matrices of UT4 and the NaCo is especially important for measurements taken in the conventional polarimetric mode (field stabilized), because the rotation of the instrument with respect to M3 causes a variation in the IP and crosstalk throughout the measurement. The technique allows us to determine the IP with an accuracy of $0.4 \%$, and constrain or determine lower or upper limits for most crosstalk components. Most notably, the UT4 $U \rightarrow V$ crosstalk is substantially larger than theory predicts. An angular offset in NaCo's half wave plate orientation is a possible source of systematic errors. We measure this offset to be $1.8^{\circ} \pm 0.5^{\circ}$.
\end{abstract}

Keywords: instrumental polarization, crosstalk, calibration scheme, polarimetric differential imaging, high contrast imaging, NAOS-CONICA, SPHERE/ZIMPOL, SPHERE/IRDIS

\section{INTRODUCTION}

Polarimetry is a powerful tool in the field of high contrast imaging and the characterization of the physical properties of circumstellar matter. For the best performance of a polarimeter, it is of paramount importance to know how much of the measured polarization comes from the science target, and how much is created by the telescope and instrument itself. Especially during observations in 'field tracking' mode, where the instrument is allowed to rotate with respect to the telescope pupil, we can no longer consider the telescope and instrument to form one system with a fixed instrumental effect on polarization. The issue of telescope/instrument induced polarization (Instrumental Polarization, IP) and transfer of one polarization state to another (crosstalk) becomes more complicated in this observing mode, because it depends on the rotation between the two components. Therefore, both telescope and instrument need to be characterized individually. IP and crosstalk can be of the order of tens of percent. ${ }^{1}$ With the commissioning of SPHERE ${ }^{2}$ during 2014, two new polarimetric imagers are put at the Nasmyth focus of Unit Telescope 3 (UT3) of the Very Large Telescope (VLT): the visible light polarimeter SPHERE/ZIMPOL ${ }^{3}$ and SPHERE/IRDIS, ${ }^{2}$ which allows for polarimetry in the near infrared (NIR). Improving the accuracy requires efficient schemes for future characterization of the contribution of both UT and instrument on the measured polarization. Improved calibration schemes are also greatly beneficial for future extremely large telescopes and facilities (e.g. E-ELT, TMT, GMT).

During the recent years, Naos-Conica ${ }^{4-6}(\mathrm{NaCo})$ has proven to be very successful with its polarimetric modes. ${ }^{7-11}$ NaCo was decommissioned and removed from VLT/UT4 in September of 2013, but will be recommissioned at VLT/UT1 during the second half of 2014. To get the best science results from both future and archival $\mathrm{NaCo}$ data, we need an accurate characterization of the UT and NaCo optics. Witzel et al. ${ }^{1}$ (2011, W11) have compared models to standard star observations to calibrate IP and crosstalk for both NaCo and

\footnotetext{
1: deboer@strw.leidenuniv.nl
} 
UT4 in Ks. De Juan Ovelar et al. (submitted) also used standard star observations to calibrate the IP in H and Ks, yet assume crosstalk between linear polarization states to be negligible. We have performed calibration measurements with $\mathrm{NaCo}$ in polarimetric mode during the last week of August 2013, using the polarized zenith skylight around sunset. Harrington et al. ${ }^{12}$ (2011, H11) have shown that if we assume the scattering of sunlight by the earth atmosphere to be dominated by single Rayleigh scattering events, we can use the skylight for accurate calibration of the telescope and instrument. We can use the a priori knowledge of the incident polarization angle, to align the system such that we can rule out specific crosstalk contributions, as is explained in Sec. 2. As pointed out by H11, this calibration light source also has the advantage that it does not require the sacrifice of valuable night time for calibration measurements.

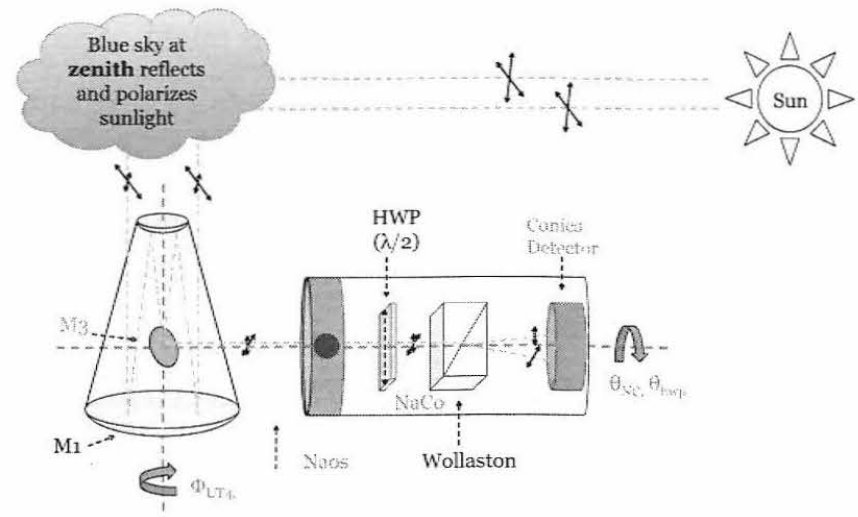

Figure 1. Schematic drawing of VLT/UT4 with NaCo at the Nasmyth focus, based on Fig. 2 of W11. The green dashed lines with green arrows indicate the rotation axes of $\Phi_{U T 4}, \theta_{N C}$ and $\theta_{H W P}$. The angles give the rotation of the optical system (or the polarization angle at the HWP), downstream from M1; NAOS; and HWP respectively.

For observations with $\mathrm{NaCo}$ in polarimetric differential imaging (PDI) mode, a Wollaston prism splits the science beam into 2 orthogonally polarized beams: the ordinary $(o)$, and extra-ordinary $(e)$ beam. A field mask behind the Wollaston prevents overlap of both beams on the Conica detector. This creates a stripe pattern of the subsequent $o$ and $e$ beams that originate from the same area in the sky (see Fig. 2). Tinbergen ${ }^{13,14}(1996)$ describes the benefits of either the double difference or double ratio method for a dual beam analyzer. Combining the 4 beams of 2 half wave plate (HWP) positions $45^{\circ}$ degrees apart, enables a correction for differences between the $o$ and $e$ beam created by transmission and efficiency imperfections of the beam splitter. The principle of double difference is taken two steps further in this study. Besides rotating the HWP with $45^{\circ}$, we also rotate $\mathrm{NaCo}$ with $90^{\circ}$ on the derotator, and UT4 with $90^{\circ}$ around the azimuth axis, as shown in Fig. 1) Each subsequent rotation allows us to correct for polarization effects induced by the instrument/telescope, downstream from the rotated optical component.

We can describe the polarization state of a beam of light with the use of the Stokes parameters: The total intensity $(I)$; the horizontal linearly polarized intensity $(Q$, with $Q<0$ for vertical polarization); the linearly polarized intensity in the $45^{\circ}$ direction ( $U$, with $U<0$ for polarization in the $135^{\circ}$ direction); and the righthanded circularly polarized intensity $(V, V<0$ for left-handed). The 4 Stokes vector components

$$
S=[I, Q, U, V]^{T},
$$

of the light receding from the $i^{\text {th }}$ optical component $S_{\text {out }}$ can be related with the incoming stokes vector $S_{\text {in }}$ according to

$$
S_{\text {out }}=M_{i} \cdot S_{\text {in }},
$$


with $M_{i}$ the $4 \times 4$ Mueller matrix for the $i^{\text {th }}$ optical component.

$$
M_{i}=\left(\begin{array}{cccc}
I \rightarrow I & Q \rightarrow I & U \rightarrow I & V \rightarrow I \\
I \rightarrow Q & Q \rightarrow Q & U \rightarrow Q & V \rightarrow Q \\
I \rightarrow U & Q \rightarrow U & U \rightarrow U & V \rightarrow U \\
I \rightarrow V & Q \rightarrow V & U \rightarrow V & V \rightarrow V
\end{array}\right) .
$$

The aim of this study is to empirically determine the components of this Mueller matrix for both UT4 ( $\left.M_{U T 4}\right)$ and $\mathrm{NaCo}\left(M_{N C}\right)$. The first $(I \rightarrow I)$ component of this matrix describes the transmittance of $i$. IP is described by the lower 3 components of the first column. Crosstalk is described by all 9 components remaining after the exclusion of the first row and the first column. The remaining 3 elements of the first row $(Q, U, V \rightarrow I)$ are best described as the influence of polarized signals on the photometry. Due to the similar design, MUT1 (NaCo's future UT) and $M_{U T 3}$ (UT SPHERE) are expected to largely resemble $M_{U T 4}$ (NaCo's previous UT), as long as we consider the same wavelength range. However, small differences might occur in time, caused by the difference of the aluminum oxide layers grown on the UT mirrors after each subsequent re-aluminization. ${ }^{15}$

In Sec. 2, we describe the underlying principles of our calibration scheme, and the default instrumental setup used. It is from this starting point that we will rotate the different optical components to perform the calibration measurements. These measurements and the data reduction are described in Sec. 3. Our results will be given in the form of Mueller matrices for both $\mathrm{NaCo}$ and UT4 in Sec. 4. The outcome is discussed together with an outlook for this study in Sec. 5 .

\section{CALIBRATION PRINCIPLES}

We aim to retrieve the individual Mueller matrices for both UT4 $\left(M_{U T 4}\right)$ and $\mathrm{NaCo}\left(M_{N C}\right)$. Combining both Mueller matrices relates the stokes vector for the light that reaches the Conica detector to the incident sky light according to:

$$
S_{\text {meas }}=T(-p) \cdot M_{N C} \cdot M_{U T 4} \cdot T(p) \cdot S_{s k y},
$$

where $T(p)$ is the rotation matrix of Eq. 37, accounting for the paralactic angle $p$ of the telescope pointing.

\subsection{Default Instrumental Setup}

Most studies $^{1,12}$ let the reference frame for the polarization angle (i.e. coordinate axes for $\pm Q$ ) be determined by the meridian. We choose the reference frame to be fixed to reflection plane of the third mirror (M3) of UT4. This choice allows us to ignore $T(p)$ in Eq. 4, but instead consider the incident $S_{s k y}$ to have changed for a different telescope pointing. We consider light to be horizontally polarized, when it is linearly polarized perpendicular to the M3 reflection plane, and vertical when the light is polarized perpendicular to both this horizontal direction and the propagation direction of the beam. We consider the following as our default setup:

- We observe sunlight that is polarized by scattering in the terrestrial atmosphere;

- UT4 is pointing at zenith, with an azimuth angle opposite to the sun: $\Phi_{U T 4}=\Phi_{\odot}+180^{\circ}=0$;

- $\mathrm{NaCo}$ is oriented such that the first mirror of Naos (M4) reflects the light in a plane perpendicular to the relection plane of $\mathrm{M} 3$. For this rotator angle of $\mathrm{NaCo}, \theta_{N C}=0$;

- The fast axis of NaCo's half wave plate retarder (HWP) is aligned with the polarization angle of the $o$ beam: $\theta_{h w p}=0$.

The Rayleigh scattered sunlight will be partially linearly polarized (to a variable, and therefore unknown degree) in the (very well known) direction orthogonal to the scattering plane in the sky (i.e. the principal plane). Skylight does not have a circular polarization component. Having $\Phi_{U T 4}=\Phi_{\odot}+180^{\circ}$ will place the M3 reflection plane orthogonal with the principal plane. This yields the following incident sky Stokes vector for the default instrumental setup:

$$
S_{s k y}=\left[I_{s k y},-Q_{s k y}, 0,0\right]^{T} .
$$


If $\left.\left(i_{o}-i_{e}\right)\right|_{\theta_{H W P}}$ is the difference between the measured intensity of the $o$ and $e$ beams for a given $\theta_{H W P}$, the classical double difference method ${ }^{14}$ can be described as:

$$
\begin{aligned}
Q_{\text {meas }}\left[\theta_{H W P}\right] & =0.5\left(\left.\left(i_{o}-i_{e}\right)\right|_{0^{\circ}}-\left.\left(i_{o}-i_{e}\right)\right|_{45^{\circ}}\right) \\
& =-Q_{\text {trans }}+Q_{U T 4}+Q_{N C},
\end{aligned}
$$

with $Q_{\text {trans }}$ the transmitted $Q_{s k y} ; Q_{U T 4}$ is the (by NaCo transmitted) UT4 induced IP, and $+Q_{N C}$ the NaCo induced IP and $\left([U, V]_{U T 4} \rightarrow Q_{N C}\right)$ crosstalk:

$$
\begin{aligned}
Q_{\text {trans }} & =M_{N C}[2,2] \times M_{U T 4}[2,2] \times Q_{\text {sky }}, \\
Q_{U T 4} & =M_{N C}[2,2] \times M_{U T 4}[2,1] \times I_{\text {sky }}, \\
Q_{N C} & =M_{N C}[2,1] \times \mathbf{I}_{U T 4}+M_{N C}[2,3] \times \mathbf{U}_{U T 4}+M_{N C}[2,4] \times \mathbf{V}_{U T 4},
\end{aligned}
$$

with $(\mathbf{I}, \mathbf{U}, \mathbf{V})_{U T 4}$ the respective $S_{\text {out }}$ parameters of UT4, incident to NaCo. $M_{U T 4}[2,2]$ and $M_{N C}[2,2]$ are related to the $Q \rightarrow Q$ components of UT4 and NaCo respectively. These components can be calibrated with a source of known Degree of Linear Polarization (DoLP $\left.=\sqrt{Q^{2}+U^{2}} / I\right)$, such as a polarized standard star. Doing so falls outside the scope of the current study, but will be included in future work. However, these components are expected to be very close to unity, which we will therefore assume below.

The corresponding intensity $I$ is determined by:

$$
I_{\text {meas }}\left[\theta_{H W P}\right]=0.5\left(\left.\left(i_{o}+i_{e}\right)\right|_{0^{\circ}}+\left.\left(i_{o}+i_{e}\right)\right|_{45^{\circ}}\right) .
$$

\subsection{Deriving UT4 Instrumental Polarization: $Q_{U T 4}$}

We extend the double difference principle by combining a measurement in the default setup $\left(=Q_{1}\right)$ with one where $\theta_{N C}=90^{\circ}\left(=Q_{2}\right)$. Just as Eq. 6 corrects for transmission difference between the $o$ and $e$ beam, a $\theta_{N C}=0,90^{\circ}$ double difference allows us to correct for NaCo's contribution to the IP (but not crosstalk), as was suggested by W11:

$$
Q_{2} \approx-Q_{\text {trans }}+Q_{U T 4}-Q_{N C} .
$$

We combine Eq. 7 and 12 with Eq. 10 to get

$$
Q_{A}=\left(Q_{1}+Q_{2}\right) / 2=-Q_{\text {trans }}+Q_{U T 4}+M_{N C}[2,3] \times \mathbf{U}_{U T 4},
$$

with

$$
\mathbf{U}_{U T 4}=M_{U T 4}[3,1] \times I_{s k y}+M_{U T 4}[3,2] \times-Q_{s k y} .
$$

To determine the IP of UT4, we can apply the same strategy of rotating the instrumental component that is to be corrected for. In this case, the strategy implies that we have to rotate the telescope itself with respect to the sky. Because we are looking at zenith, we will still point at the same part of the sky after changing the telescope azimuth to $\Phi_{U T 4}=90^{\circ}$. Since we decided to use the scattering plane of M3 as a reference, this changes the incident stokes vector from Eq. 5 to $S_{s k y}=\left[I_{s k y}, Q_{s k y}, 0,0\right]^{T}$. Repeating the two previous measurements for the new azimuth position gives:

$$
\begin{aligned}
Q_{3} & =Q_{\text {trans }}+Q_{U T 4}+Q_{N C} \\
Q_{4} & =Q_{\text {trans }}+Q_{U T 4}-Q_{N C} \\
Q_{B} & =\left(Q_{3}+Q_{4}\right) / 2=Q_{\text {trans }}+Q_{U T 4}-M_{N C}[2,3] \times \mathbf{U}_{U T 4} .
\end{aligned}
$$

The changing sign of $\mathbf{U}_{U T 4}$ is caused by the changing sign of $Q_{s k y}$, and the assumption that $M_{U T 4}[3,1]=0$, as is proposed by W11 and H11. This allows us to retrieve the UT4 induced IP according to:

$$
Q_{U T 4}=\left(Q_{A}+Q_{B}\right) / 2
$$




\subsection{From measurements to matrix components}

We assume our pointing to be perfect, meaning that our light incident to our telescope is either $S_{\text {sky }}=$ $[I, \pm Q, 0,0]^{T}$ (Aug. 31), or $S_{s k y}=[I, 0, \pm U, 0]^{T}$ (Sep. 1). In Tabs. 3 and 5 of Appendix B, we list the different transmission, instrumental polarization and crosstalk components measured with the setups listed in Tabs. 2 and 4 of Appendix B. Just as we did in the previous section for $Q_{U T 4}$, we can use the sign changes of the listed components for different instrumental setups. This sign change allows us to isolate the components by adding and subtracting the individual measurements. From Tab. 3, we can find that from the $16 X_{s k y} \rightarrow X_{U T 4} \rightarrow X_{N C}$ components of the $Q_{1-4}$ measurements, we can isolate 8 pairs of components:

$$
\begin{aligned}
(I \rightarrow I \rightarrow Q, U)+(I \rightarrow V \rightarrow Q, U) & =\left[+Q, U_{1}-Q, U_{2}+Q, U_{3}-Q, U_{4}\right] / 4, \\
(I \rightarrow Q \rightarrow Q, U)+(I \rightarrow U \rightarrow Q, U) & =\left[+Q, U_{1}+Q, U_{2}+Q, U_{3}+Q, U_{4}\right] / 4, \\
(Q \rightarrow I \rightarrow Q, U)+(Q \rightarrow V \rightarrow Q, U) & =\left[-Q, U_{1}+Q, U_{2}+Q, U_{3}-Q, U_{4}\right] / 4, \\
(Q \rightarrow Q \rightarrow Q, U)+(Q \rightarrow U \rightarrow Q, U) & =\left[-Q, U_{1}-Q, U_{2}+Q, U_{3}+Q, U_{4}\right] / 4,
\end{aligned}
$$

where Eq. 20 is the same Eq. 18 and is what we called the UT4 $I \rightarrow Q$ instrumental polarization. For each equation above, $Q, U$ means either $Q$ or $U$. Similarly, from the 16 measurements listed in Tab. 5 we can isolate the following 8 pairs of components:

$$
\begin{aligned}
(I \rightarrow I \rightarrow Q, U)+(I \rightarrow V \rightarrow Q, U) & =\left[+Q, U_{5}-Q, U_{6}+Q, U_{7}-Q, U_{8}\right] / 4, \\
(I \rightarrow Q \rightarrow Q, U)+(I \rightarrow U \rightarrow Q, U) & =\left[+Q, U_{5}+Q, U_{6}+Q, U_{7}+Q, U_{8}\right] / 4, \\
(U \rightarrow I \rightarrow Q, U)+(U \rightarrow V \rightarrow Q, U) & =\left[+Q, U_{5}-Q, U_{6}-Q, U_{7}+Q, U_{8}\right] / 4, \\
(U \rightarrow Q \rightarrow Q, U)+(U \rightarrow U \rightarrow Q, U) & =\left[+Q, U_{5}+Q, U_{6}-Q, U_{7}-Q, U_{8}\right] / 4 .
\end{aligned}
$$

\section{OBSERVATIONS AND DATA REDUCTION}
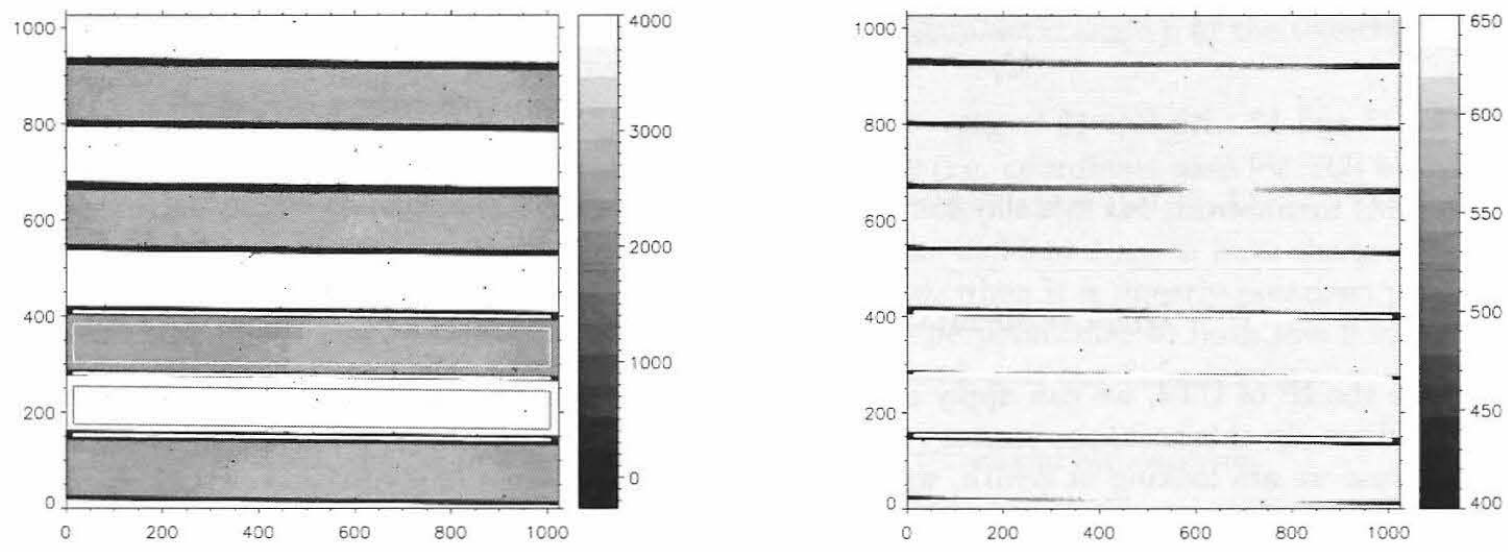

Figure 2. Left: Example of the $o$ and $e$ stripe pattern accross the detector, with dark masked areas in between. The mean pixel values in the blue and yellow boxes are respectively $i_{o}$ and $i_{e}$ in Eq. 6 . Right: NaCo detector image, with a dynamic range chosen to show the masked regions. This dynamic range shows that stray light has contaminated both the masked and unmasked regions alike. The 3 green boxes in the masked areas are used to determine the stray light correction of Sec. 3.3.

\subsection{IP and crosstalk calibration observations}

We have observed the sky light just before and after sunset at both the $31^{\text {st }}$ of August, and the $1^{\text {st }}$ of September of 2013. All our observations have been performed in the $\mathrm{H}$ band, have (NDIT $=$ ) 2 exposures of 4 seconds. Appendix B lists the observations and their changes with respect to the default instrumental setup of Sec. 2.1. 
The $31^{\text {st }}$ of August, we have observed the sky with an incident polarization angle at either $0^{\circ}$ or $90^{\circ}$ with respect to the reference frame of UT4. We observed around sunset, when the intensity of the sky light decreases quickly, while the DoLP increases, even beyond the $90^{\circ}$ scattering angle of sunset. For each UT4/NaCo position, $Q$ and $U$ are measured using a set of 4 HWP positions according to the double difference method of Eq. 6: $\theta_{H W P}=[0,45,22.5,67.5]$. These sets of HWP angles are repeated for all 4 possible UT4/NaCo combinations, rotating either or both UT4 and $\mathrm{NaCo}$ with $90^{\circ}$. The HWP set is repeated for the first (= default) UT4/NaCo position to allow us to correct for changing sky conditions. The resulting 5 HWP sets are listed in Tab. 2.

The evening of the $1^{\text {st }}$ of September, the sky polarization angle was at either $+45^{\circ}$ or $-45^{\circ}$ compared to the M3 reflection plane. This time, we have performed the entire sequence two times, for a better correction of the change of incident polarization and intensity. The observations taken this evening are listed in Tab. 4 .

During the observations, the sun moved accross the sky. The changing solar azimuth position creates a small uncertainty in the polarization angle of $\Delta \Phi_{\odot}=1^{\circ}$. The decreasing altitude, accounts for a large change in incident DoLP, as is discussed in Sec. 3.3.

\subsection{HWP angle offset calibration observations}

On the $1^{\text {st }}$ of September 2013, we have observed the Zenith sky in the default instrumental setup, for 16 different HWP angles, with intervals of $11.25^{\circ}$.

\subsection{Data reduction}

After we take the mean of the two individual exposures (DITS) per HWP angle, A thermal background or dark measurement is subtracted from this mean sky frame. The sky frame is divided by a normalized sky flatfield, the latter taken without the Wollaston. The lefthand image of Fig. 2 shows the resulting sky frame, from which we select one large area $(994 \times 80$ pixels) in both an $o$ beam stripe (blue box), and an $e$ beam stripe (yellow box).

Stray light is visible as a brighter band running diagonally accross the detector, from the top (right from center) to the bottom center. The righthand image shows the same frame, for the value range of $525 \pm 125$ counts, which are the mean values of the areas masked by the Wollaston mask of NaCo. Ideally, the values of the masked regions should on average be zero after dark subtraction. However, we can clearly see the same stray light band run accross the masked region. To correct for this stray light, each pixel column of the boxed areas of interest are corrected in the following way: We determined the mean value of five masked pixels above, and five masked pixels below both the $o$ and $e$ boxes of interest (indicated by the 3 green boxed areas in the righthand image of Fig. 2), and subtract this value from the corresponding pixel columns of the $o$ and $e$ boxes.

We correct for dead or hot pixels by replacing each pixel which has a value deviating from the mean with more than 3 sigma, with the median value of its surrounding pixels. $i_{o}$ and $i_{e}$ of Eq. 6 are in fact the mean pixel value for the $o$ and $e$ box respectively.

\section{Changing sky conditions}

During the cycle of observations the solar altitude decreases, leaving neither the intensity, nor the polarization of the sky light constant. For this study we have used the simple assumption of a linearly increasing DoLP with time, which is the consequence of single Rayleigh scattering. ${ }^{12}$ The repetition of an identical set of measurements at the beginning and at the end of the cycle allows us to determine the slope of the polarized fraction. We have corrected all measured values for the average increase of the polarized fraction of datapoints with identical instrumental setup. Fig. 3 shows the fractional polarization for the measurements taken on the $1^{\text {st }}$ of September, before and after correction for changing sky polarization.

\section{Double Difference: 2 half wave plate angles}

The double difference values are determined according to Eq. 6, and divided by $I$. In Fig. 4, we plot the absolute values of the double difference divided by $I$ against the measurement time. Fig. 4 plots the absolute value to illustrate the fluctuations in the measured values of $(Q, U)_{\text {meas }} / I_{\text {meas }}$ for the different instrumental setups (described in the legend). Keep in mind that our equations of Sec. 2 do not use the absolute values. 

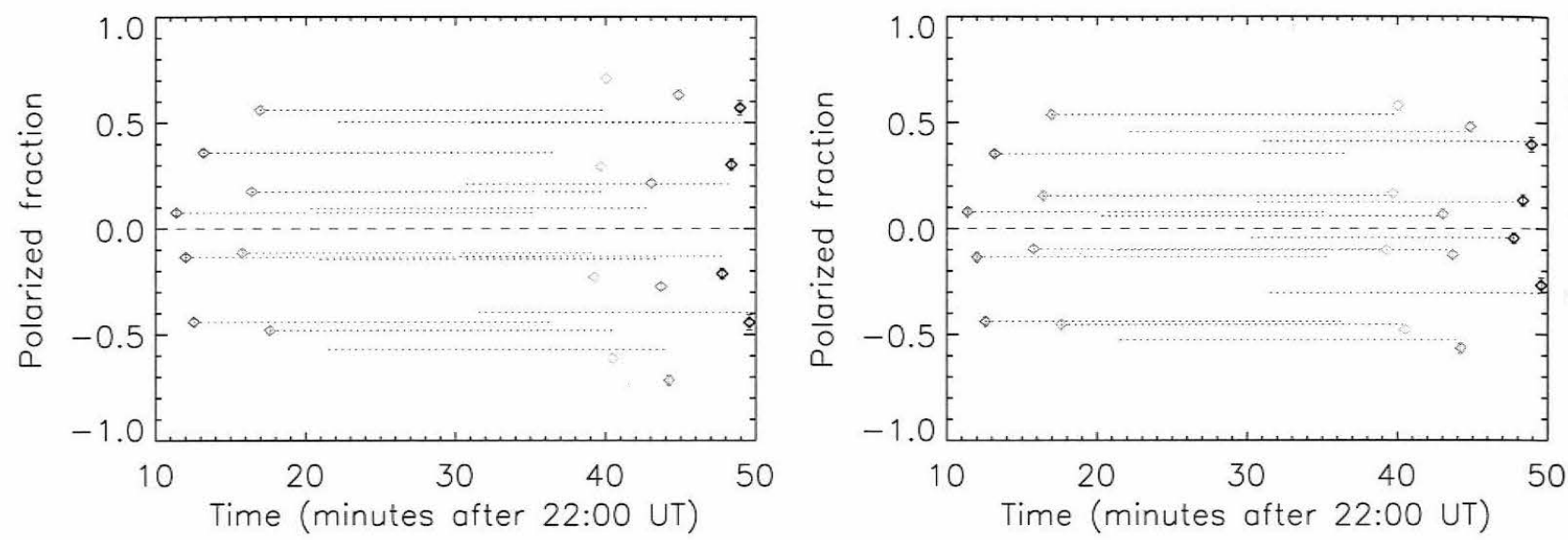

Figure 3. Fractional polarization of the September 1 measurements (Tab. 4) plotted against time (minutes after 22:00 UTC $=6$ p.m. local time) Left: Before correcting for the increasing sky polarization. Right: After correcting for increasing sky polarization. The horizontal dotted lines connect the datapoints with the same instrumental setup. Given ideal conditions (i.e. time constant $S_{i n}$ ), two datapoints on either side of the horizontal lines, would have the same fractional polarization. The colors represent the different instrumental setups, explained in the legends of Fig. 4
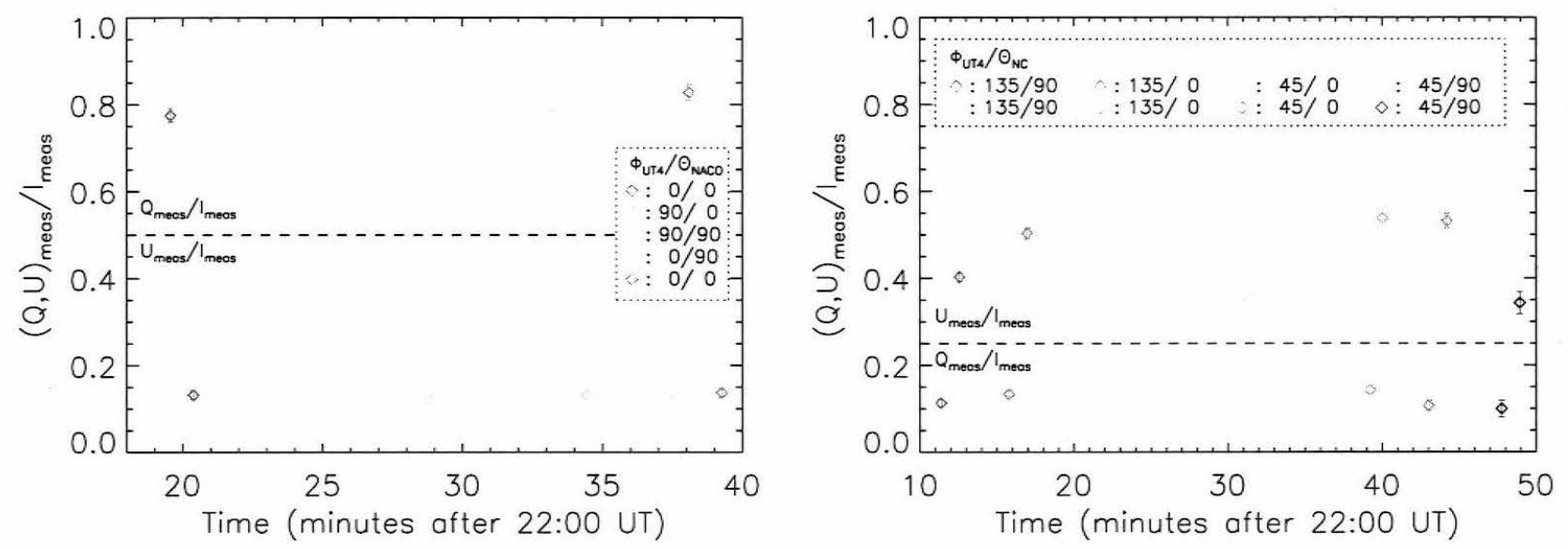

Figure 4. The absolute values of the classical double difference: combining 2 measurement with HWP angles $45^{\circ}$ apart to determine $|Q, U|_{\text {meas }} / I_{\text {meas }}$. Left: August 31 measurements with $S_{s k y}=[I, \pm Q, 0,0]^{T}$. Without exception: $|Q / I|_{\text {meas }}>$ 0.5 , and $|U / I|_{\text {meas }}<0.5$. Right: September 1 measurement with $S_{\text {sky }}=[I, 0, \pm U, 0]^{T}$. Without exception: $|Q / I|_{\text {meas }}<$ 0.25 , and $|U / I|_{\text {meas }}>0.25$.

\section{Double Difference: 2 NaCo derotator angles}

The first $4(Q / I)_{\text {meas }}(>0.5)$ values of the $S_{\text {sky }}=[I, \pm Q, 0,0]^{T}$ measurements of Fig. 4 are in fact $Q_{1-4}$ of Sec. 2.2, albeit not in the same order. Due to our choice of reference frame, $Q_{A, B}$ of Eqs. 13 and 17 are a second example of the double difference technique. This time, instead of combining different HWP angles, we combine measurements taken before and after a rotation of $\mathrm{NaCo}$ of $90^{\circ}$. With this double difference we cancel out $M_{N C}[2,1] \times \mathbf{I}_{U T 4}$ and $M_{N C}[2,4] \times \mathbf{V}_{U T 4}$ of Eq. 10 .

\section{RESULTS}

\subsection{NaCo HWP offset}

The offset between the fast axis of NaCo's half wave plate and the angle given in the driver software and image headers has been much debated in literature. Witzel et al. ${ }^{16}$ (2010, W10) report that the angle between the 


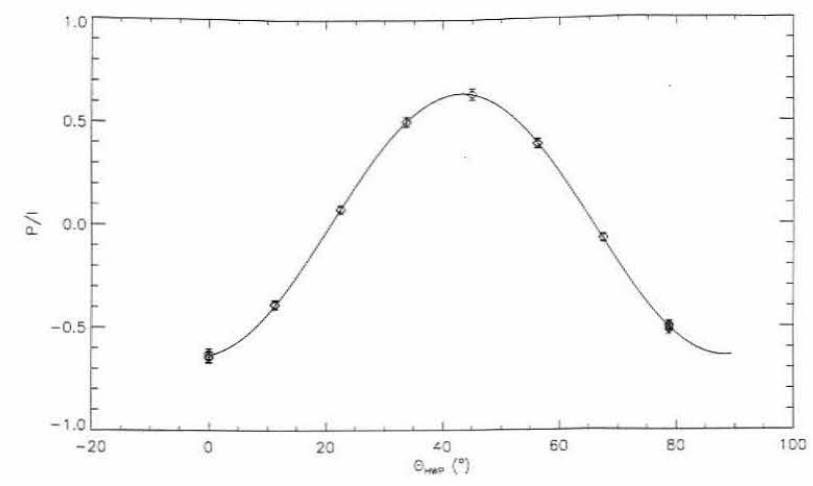

Figure 5. Polarized fraction plotted against the angle of the half wave plate, as given by the FITS header. The solid line is a best fit $-\cos \left(2 \theta_{H W P}+\phi_{H W P}\right)$, with an offset angle of $\phi_{H W P}=1.8^{\circ} \pm 0.5^{\circ}$.

reference system and the HWP fast axis was as large as $-6.6 \pm 0.2^{\circ}$. According to the $2009 \mathrm{NaCo}$ intervention report, the zero-encoder position is "set to zero". There is no mention in the report what the angular change is with respect to the pre-2009 position. However, W11 state that the difference of the revision is this very $6.6^{\circ}$. In other words, it does not have to be taken into account for measurements after 2009.

Various authors have used the warning by W10 as an argument to take a HWP offset into account. Garufi et al. ${ }^{8}$ (2013) and Avenhaus et al. ${ }^{11}$ (2014) fit the offset angle for each dataset. Our calibration scheme is based on the assumption of a known angle of the sky polarization. For large HWP angular offsets we would need to correct Eqs. 19 to 26 . We therefore calibrated the HWP offset by measuring the polarized fraction for different HWP angles, with $\Delta \theta_{H W P}=11.25$, which we plotted in Fig. 5. We have fitted the data to:

$$
A_{0} \cos \left(\theta_{H W P}-\phi_{H W P}\right)=A_{1} \sin \left(\theta_{H W P}\right)+A_{2} \cos \left(\theta_{H W P}\right),
$$

where $A_{1}$ and $A_{2}$ are determined with a linear regression. From this, we can constrain the constants $A_{0}$ and $\phi_{H W P}$ according to:

$$
\begin{aligned}
(Q / I)_{s k y} \approx A_{0} & =\sqrt{A_{1}^{2}+A_{2}^{2}}, \\
\phi_{H W P} & =\arctan \left(A_{1} / A_{2}\right) .
\end{aligned}
$$

The best fit yields an offset of $\phi_{H W P}=1.8^{\circ} \pm 0.5^{\circ}$, where the error is dominated by the uncertainty in the solar azimuth angle of $1^{\circ}$. This HWP offset is responsible for a measurement error $<0.0016$, much smaller than the random error in our measurements (see Tab. 1). Based on this result, we have ignored any error caused by the HWP offset.

\subsection{Matrix components}

We have listed the measured results of Eqs. 19 to 26 under "Result" in Tab. 1. As can be seen from the equations under "Component", the result reflects de facto multiple matrix components. However, the $M_{U T 4}$ and/or $M_{N C}$ components which are most constrained by the result, are listed in the column "Constraining". This does not always mean that this matrix component is the same as the measured result. For example, to determine $M_{N C}[2,4]$ from the result of Eq. 25, the result has to be divided by both $U_{s k y}$ and $M_{U T 4}[4,3]$.

\subsubsection{UT4 Mueller matrix components}

The "Constraining' column of Tab. 1 only holds one value for $M_{U T 4}$, namely the $[2,1]$ component, determined from the mean of Eqs. 20 and 24. We know that due to the symmetry in M1 and M2, neither of these mirrors should contribute to $M_{U T 4}$. We can therefore consider $M_{U T 4}=M_{M 3}$. For a one mirror matrix, we can assume that it is symmetric around the diagonal, and compute the remaining values using the Fresnel equations, ${ }^{1,15}$ 
Table 1. Results of Eqs. 19 to 26, based on sky observations taken at the 31st of August (3rd column) and the 1st of September (5th column), 2013.

\begin{tabular}{|l|r|l|l|r|l|}
\hline Component & Eq. & Result & Eq. & Result & Constraining \\
\hline \hline$(I \rightarrow I \rightarrow Q)+(I \rightarrow V \rightarrow Q)$ & 19 & $-0.004 \pm 0.008$ & 23 & $-0.008 \pm 0.004$ & $M_{N C}[2,1]$ \\
\hline$(I \rightarrow I \rightarrow U)+(I \rightarrow V \rightarrow U)$ & 19 & $-0.003 \pm-0.008$ & 23 & $-0.007 \pm 0.004$ & $M_{N C}[3,1]$ \\
\hline$(I \rightarrow Q \rightarrow Q)+(I \rightarrow U \rightarrow Q)$ & 20 & $-0.006 \pm 0.008$ & 24 & $-0.013 \pm 0.004$ & $M_{U T 4}[2,1]$ \\
\hline$(I \rightarrow Q \rightarrow U)+(I \rightarrow U \rightarrow U)$ & 20 & $-0.016 \pm 0.008$ & 24 & $0.010 \pm 0.004$ & $M_{U T 4}[2,1] * M_{N C}[3,2]$ \\
\hline$(Q \rightarrow I \rightarrow Q)+(Q \rightarrow V \rightarrow Q)$ & 21 & $-0.008 \pm 0.008$ & & & not significant \\
\hline$(Q \rightarrow I \rightarrow U)+(Q \rightarrow V \rightarrow U)$ & 21 & $-0.0003 \pm 0.008$ & & & not significant \\
\hline$(Q \rightarrow Q \rightarrow U)+(Q \rightarrow U \rightarrow U)$ & 22 & $-0.114 \pm 0.008$ & & & $M_{N C}[3,2]$ \\
\hline$(U \rightarrow I \rightarrow Q)+(U \rightarrow V \rightarrow Q)$ & & & 25 & $-0.009 \pm 0.004$ & $M_{N C}[2,4]$ \\
\hline$(U \rightarrow I \rightarrow U)+(U \rightarrow V \rightarrow U)$ & & & 25 & $0.074 \pm 0.004$ & $M_{N C}[3,4]$ \\
\hline$(U \rightarrow Q \rightarrow Q)+(U \rightarrow U \rightarrow Q)$ & & & 26 & $-0.107 \pm 0.004$ & $M_{N C}[2,3]$ \\
\hline
\end{tabular}

with the refrective indices for aluminum in $\mathrm{H}$ band being $R_{\perp}=0.98308 ; R_{\|}=0.96644$ and its reflection phase for a $45^{\circ}$ angle of incidence are $\phi_{\perp}=-175.108^{\circ} ; \phi_{\|}=-170.215^{\circ} *$. The resulting Mueller matrix for $U T 4$ is:

$$
M_{U T 4}=\left(\begin{array}{cccc}
1 & -0.010 \pm 0.004 & 0 & 0 \\
-0.010 \pm 0.004 & 1 & 0 & 0 \\
0 & 0 & -0.996 & -0.0853 \\
0 & 0 & 0.0853 & -0.996
\end{array}\right) .
$$

The black values in Eq. 30 represent the ones determined from the results in Tab. 1, whereas the blue values are computed from the Fresnel equations.

\subsubsection{NaCo Mueller matrix components}

Once we have determined $M_{U T 4}$, we can determine the values from $M_{N C}$. Because NaCo IP and crosstalk is not determined by multiple reflections and transmissions, we can no longer assume the matrix to be symmetric. Therefore, we have assumed the values for the first row of $M_{N C}$, which hardly influence our measurements, and ignored the last row, which $\mathrm{NaCo}$ cannot measure.

$$
M_{N C}=\left(\begin{array}{cccc}
1 & 0 & 0 & 0 \\
-0.006 \pm 0.004 & 1 & 0.160 \pm 0.068 & -0.170 \pm 0.058 \\
-0.005 \pm 0.004 & 0.147 \pm 0.071 & -0.662 \pm 0.338 & 1.31 \\
- & - & - & -
\end{array}\right)
$$

Once again, the black values in Eq. 31 are determined from the results listed in Tab. 1. The red values are components that still have to be changed, due to the considerations below. The large uncertainties on the $[2,3],[2,4]$ and $[3,4]$ components are caused by the large uncertainty of our $U_{s k y}$, by which we have to divide the results of Tab. 1 to determine the corresponding matrix components. This uncertainty is in turn created by our lack of knowledge of component $[3,3]$. If we assume $M_{N C}[3,3]=1$, then $(U / I)_{s k y}=M_{U T 4}[3,3] \times 0.442=$ $0.996 \times 0.442=0.440$, but this is not likely to be the case. Therefore, we have used this value as a lower limit of $(U / I)_{s k y}$, and used the highes $Q_{\text {meas }}$ value of 0.9 as an upperlimit: $(U / I)_{s k y}=0.670 \pm 0.230$, and $M_{N C}[3,3]=0.662 \pm 0.338$.

We are left with a non-physical value for $M_{N C}[3,4]=($ result of Eq. 25$) /(U / I)_{\text {sky }} /[4,3]_{U T}$, which becomes $0.074 / 0.662 / 0.0853=1.31$. This means that there must be an error in our assumptions, both of which would be quite surprising:

- $M_{U T 4}[1,3] \neq 0$

${ }^{*}$ Refrective indices and reflection phases are obtained from www. RefractiveIndex.INFO. 
- $M_{U T 4}[4,3]>>0.0853$

Besides bringing down the value for $M_{N C}[2,4]$, changing either would impact at least and $M_{N C}[2,4], M_{N C}[2,2]$, and $M_{U T 4}[3,3]$. Finding the optimal solution is best done iteratively, which we leave for future studies. Below, we discuss a very coarse analysis of either proposed solution for the non-physicality of the $V \rightarrow U$ crosstalk of Naco.

\section{Substantial UT4 $U \rightarrow I$ component}

If we don't change any of the other components, the most extreme case, with $M_{U T 4}[1,3]=1$ yields:

$$
M_{N C}[3,4]=(0.074+0.670 \times-0.005) /(0.670 \times 0.085)=1.240 .
$$

Since $M_{N C}[3,4]>1$, we can conclude that this option does not solve the problem.

\section{Larger UT4 $U \rightarrow V$ crosstalk}

A solution for:

$$
M_{N C}[3,4]=0.074 /\left(0.670 \times M_{U T 4}[4,3]\right)<1,
$$

can be found for $0.11<M_{U T 4}[4,3]<1$, which yields $1>M_{N C}[3,4]>0.11$. This provides lower limits for the new Mueller matrices:

$$
M_{U T 4}=\left(\begin{array}{cccc}
1 & -0.010 \pm 0.004 & 0 & 0 \\
-0.010 \pm 0.004 & 1 & 0 & 0 \\
0 & 0 & >-0.994 & <-0.110 \\
0 & 0 & >0.110 & >-0.994
\end{array}\right)
$$

and

$$
M_{N C}=\left(\begin{array}{cccc}
1 & 0 & 0 & 0 \\
-0.006 \pm 0.004 & 0.983 \pm 0.017 & 0.161 \pm 0.068 & >-0.122 \\
-0.005 \pm 0.004 & 0.147 \pm 0.071 & -0.662 \pm 0.338 & >0.110 \\
- & - & - & -
\end{array}\right)
$$

The $M_{N C}[2,2]$ value is determined by normalizing the last three values of the second row according to $[2,2]=$ $\sqrt{[2,3]^{2}+[2,4]^{2}}$. In the same way, we have determined $M_{U T 4}[3,3]$.

\section{DISCUSSION AND OUTLOOK}

We present the preliminary constraints on the Mueller matrices for UT4 and NaCo. These matrix values are obtained by observing the polarized blue sky at zenith, while rotating both UT4 and $\mathrm{NaCo}$ with $90^{\circ}$ angles, as described in Sec. 2. Despite the large error bars for some matrix components of Eqs. 34 and 35, or that we could only determine upper or lower limits for other components, this study is an important first step for a more accurate determination of the effects of the telescope and $\mathrm{NaCo}$ on the measured polarization. We find that the UT4 $U \rightarrow V$ crosstalk is substantially larger than theory predicts, which might be explained by the existance of an aluminium-oxide coating of M3. ${ }^{15}$

To the best or our knowledge, for neither $\mathrm{NaCo}$, nor UT4, complete Mueller matrices are published for the $\mathrm{H}$ band. W11 have determined the full $M_{N C}$ for the Ks band, with the use of standard star observations. The advantage of our calibration method is that it can be performed without using valuable night time. De Juan Ovelar et al. (submitted) determined the IP for both $\mathrm{H}$ and $\mathrm{Ks}$ band. They find $I \rightarrow Q_{N C}=-0.024$, which is 4 times higher than our value of -0.006 , but their $I \rightarrow U_{N C}=-0.005$ is in good agreement with our value. The difference between their $\mathrm{H}$ and Ks values teach us that a direct comparison between our $M_{N C}(\mathrm{H})$ and the $M_{N C}(\mathrm{Ks})$ of W11 is not valid. The mueller matrix appears to be too color dependant to do so.

For our matrices to be usefull for the correction of astrophysical data, we need to determine especially $M_{N C}[3,3]$ with higher precision. We intend to constrain this value further with the use of standard star observations. Furthermore, H11 have demonstrated that a normal non-linear least-squares minimalization can be applied to determine the Mueller matrix values. The next step in our study will be to apply this least squares method as a further refinement of our calibration. 


\section{Acknowledgements}

JdB acknowledges ESO for its support provided with the studentship program. We furthermore thank ESO staff of the Paranal Observatory for their support during the observations, especially Fernando Salgado.

\section{APPENDIX A. ROTATIONAL (A)SYMMETRY OF MUELLER MATRIX COMPONENTS}

Whether rotating an element by $90^{\circ}$ changes the sign of the IP or crosstalk component, depends on whether the rotation occurs upstream or downstream from the reference frame. Rotating the component fixed to the reference plane is equal to rotating the polarization angle of the incident light (i.e. upstream rotation):

$$
M_{\text {upstream_rot }=90^{\circ}}=M \cdot T\left(90^{\circ}\right)=\left(\begin{array}{ccc}
+ & - & - \\
+ & - & - \\
+ & - & + \\
+ & - & -
\end{array}\right),
$$

with $T\left(90^{\circ}\right)$ the rotation matrix: ${ }^{1}$

$$
T(p)=\left(\begin{array}{cccc}
1 & 0 & 0 & 0 \\
0 & \cos (2 p) & \sin (2 p) & 0 \\
0 & -\sin (2 p) & \cos (2 p) & 0 \\
0 & 0 & 0 & 1
\end{array}\right)
$$

A + sign in Eq. 36 indicates that the matrix component does not change sign with rotation whereas a - sign means that the matrix component does change sign. Notice that $I_{i n}$ and $V_{i n}$ do not change with rotation. Therefore, the signs of the matrix components of the first and last column remain unchanged.

Rotating the optical system downstream from the reference frame gives a different behaviour, because we need an additional $T\left(-90^{\circ}\right)$ in the equation to get back to our initial reference frame. In this case, the change of sign occurs for the elements where a Stokes component insensitive to rotation $(I, V)$ is transformed in a Stokes component that is $(Q, U)$, or vice versa:

$$
M_{\text {downstream_rot }=90^{\circ}}=T\left(-90^{\circ}\right) \cdot M \cdot T\left(90^{\circ}\right)\left(\begin{array}{cccc}
+ & - & - & + \\
- & + & + & - \\
- & + & + & - \\
+ & - & - & +
\end{array}\right) .
$$

\section{APPENDIX B. OBSERVATION TABLES}


Table 2. Sky observations taken at the 31st of August 2013. All coordinates are given as changes with respect to the default instrumental setup of Sec. 2.1 .

\begin{tabular}{|c|c|c|c|c|}
\hline Universal Time & $\Phi_{U T 4}\left(^{\circ}\right)$ & $\theta_{N C}\left(^{\circ}\right)$ & $\theta_{H W P}\left(^{\circ}\right)$ & $\left(Q, U_{1-4}\right.$ \\
\hline \hline $22: 19: 34.2$ & 0 & 0 & 0 & $Q_{1}$ \\
$22: 20: 00.8$ & 0 & 0 & 45 & $Q_{1}$ \\
$22: 20: 22.9$ & 0 & 0 & 22.5 & $U_{1}$ \\
$22: 20: 49.5$ & 0 & 0 & 67.5 & $U_{1}$ \\
$22: 23: 18.1$ & 90 & 0 & 0 & $Q_{3}$ \\
$22: 23: 55.9$ & 90 & 0 & 45 & $Q_{3}$ \\
$22: 24: 29.1$ & 90 & 0 & 22.5 & $U_{3}$ \\
$22: 25: 06.8$ & 90 & 0 & 67.5 & $U_{3}$ \\
$22: 27: 39.0$ & 90 & 90 & 0 & $Q_{4}$ \\
$22: 28: 16.6$ & 90 & 90 & 45 & $Q_{4}$ \\
$22: 28: 49.0$ & 90 & 90 & 22.5 & $U_{4}$ \\
$22: 29: 27.3$ & 90 & 90 & 67.5 & $U_{4}$ \\
$22: 33: 11.6$ & 0 & 90 & 0 & $Q_{2}$ \\
$22: 33: 51.6$ & 0 & 90 & 45 & $Q_{2}$ \\
$22: 34: 24.8$ & 0 & 90 & 22.5 & $U_{2}$ \\
$22: 35: 02.5$ & 0 & 90 & 67.5 & $U_{2}$ \\
$22: 38: 05.2$ & 0 & 0 & 0 & $Q_{1}$ \\
$22: 38: 42.8$ & 0 & 0 & 45 & $Q_{1}$ \\
$22: 39: 16.0$ & 0 & 0 & 22.5 & $U_{1}$ \\
$22: 39: 54.8$ & 0 & 0 & 67.5 & $U_{1}$ \\
\hline
\end{tabular}

Table 3. A list of the compenents we measure with the different instrumental setups of Tab. 2. All components are listed in the form $X_{s k y} \rightarrow X_{U T 4} \rightarrow X_{N C}$, but we do not state the subscripts.

$$
\begin{array}{cllll}
Q_{1}= & +(I \rightarrow I \rightarrow Q) & +(I \rightarrow Q \rightarrow Q) & +(I \rightarrow U \rightarrow Q) & +(I \rightarrow V \rightarrow Q) \\
& -(Q \rightarrow I \rightarrow Q) & -(Q \rightarrow Q \rightarrow Q) & -(Q \rightarrow U \rightarrow Q) & -(Q \rightarrow V \rightarrow Q) \\
Q_{2}= & -(I \rightarrow I \rightarrow Q) & +(I \rightarrow Q \rightarrow Q) & +(I \rightarrow U \rightarrow Q) & -(I \rightarrow V \rightarrow Q) \\
& +(Q \rightarrow I \rightarrow Q) & -(Q \rightarrow Q \rightarrow Q) & -(Q \rightarrow U \rightarrow Q) & +(Q \rightarrow V \rightarrow Q) \\
Q_{3}= & +(I \rightarrow I \rightarrow Q) & +(I \rightarrow Q \rightarrow Q) & +(I \rightarrow U \rightarrow Q) & +(I \rightarrow V \rightarrow Q) \\
& +(Q \rightarrow I \rightarrow Q) & +(Q \rightarrow Q \rightarrow Q) & +(Q \rightarrow U \rightarrow Q) & +(Q \rightarrow V \rightarrow Q) \\
Q_{4}= & -(I \rightarrow I \rightarrow Q) & +(I \rightarrow Q \rightarrow Q) & +(I \rightarrow U \rightarrow Q) & -(I \rightarrow V \rightarrow Q) \\
& -(Q \rightarrow I \rightarrow Q) & +(Q \rightarrow Q \rightarrow Q) & +(Q \rightarrow U \rightarrow Q) & -(Q \rightarrow V \rightarrow Q) \\
& & & & \\
U_{1}= & +(I \rightarrow I \rightarrow U) & +(I \rightarrow Q \rightarrow U) & +(I \rightarrow U \rightarrow U) & +(I \rightarrow V \rightarrow U) \\
& -(Q \rightarrow I \rightarrow U) & -(Q \rightarrow Q \rightarrow U) & -(Q \rightarrow U \rightarrow U) & -(Q \rightarrow V \rightarrow U) \\
U_{2}= & -(I \rightarrow I \rightarrow U) & +(I \rightarrow Q \rightarrow U) & +(I \rightarrow U \rightarrow U) & -(I \rightarrow V \rightarrow U) \\
& +(Q \rightarrow I \rightarrow U) & -(Q \rightarrow Q \rightarrow U) & -(Q \rightarrow U \rightarrow U) & +(Q \rightarrow V \rightarrow U) \\
U_{3}= & +(I \rightarrow I \rightarrow U) & +(I \rightarrow Q \rightarrow U) & +(I \rightarrow U \rightarrow U) & +(I \rightarrow V \rightarrow U) \\
& +(Q \rightarrow I \rightarrow U) & -(Q \rightarrow Q \rightarrow U) & -(Q \rightarrow U \rightarrow U) & +(Q \rightarrow V \rightarrow U) \\
U_{4}= & -(I \rightarrow I \rightarrow U) & +(I \rightarrow Q \rightarrow U) & +(I \rightarrow U \rightarrow U) & -(I \rightarrow V \rightarrow U) \\
& -(Q \rightarrow I \rightarrow U) & +(Q \rightarrow Q \rightarrow U) & +(Q \rightarrow U \rightarrow U) & -(Q \rightarrow V \rightarrow U)
\end{array}
$$


Table 4. Sky observations taken at the 1st of September, 2013.

\begin{tabular}{|c|c|c|c|c|}
\hline Universal Time & $\Phi_{U T 4}\left(^{\circ}\right)$ & $\theta_{N C}\left(^{\circ}\right)$ & $\theta_{H W P}\left({ }^{\circ}\right)$ & $\left(Q, U_{5-8}\right.$ \\
\hline \hline $22: 11: 23.2$ & 135 & 90 & 0 & $Q_{8}$ \\
$22: 12: 00.7$ & 135 & 90 & 45 & $Q_{8}$ \\
$22: 12: 33.9$ & 135 & 90 & 22.5 & $U_{8}$ \\
$22: 13: 12.1$ & 135 & 90 & 67.5 & $U_{8}$ \\
$22: 15: 47.4$ & 135 & 0 & 0 & $Q_{7}$ \\
$22: 16: 24.6$ & 135 & 0 & 45 & $Q_{7}$ \\
$22: 16: 57.4$ & 135 & 0 & 22.5 & $U_{7}$ \\
$22: 17: 37.4$ & 135 & 0 & 67.5 & $U_{7}$ \\
$22: 20: 19.7$ & 45 & 0 & 0 & $Q_{5}$ \\
$22: 20: 56.9$ & 45 & 0 & 45 & $Q_{5}$ \\
$22: 21: 31.8$ & 45 & 0 & 22.5 & $U_{5}$ \\
$22: 22: 11.0$ & 45 & 0 & 67.5 & $U_{5}$ \\
$22: 30: 15.5$ & 45 & 90 & 0 & $Q_{6}$ \\
$22: 30: 42.4$ & 45 & 90 & 45 & $Q_{6}$ \\
$22: 31: 04.5$ & 45 & 90 & 22.5 & $U_{6}$ \\
$22: 31: 31.2$ & 45 & 90 & 67.5 & $U_{6}$ \\
$22: 35: 27.0$ & 135 & 90 & 0 & $Q_{8}$ \\
$22: 35: 53.8$ & 135 & 90 & 45 & $Q_{8}$ \\
$22: 36: 15.8$ & 135 & 90 & 22.5 & $U_{8}$ \\
$22: 36: 42.5$ & 135 & 90 & 67.5 & $U_{8}$ \\
$22: 39: 13.7$ & 135 & 0 & 0 & $Q_{7}$ \\
$22: 39: 40.5$ & 135 & 0 & 45 & $Q_{7}$ \\
$22: 40: 02.5$ & 135 & 0 & 22.5 & $U_{7}$ \\
$22: 40: 29.3$ & 135 & 0 & 67.5 & $U_{7}$ \\
$22: 43: 02.0$ & 90 & 0 & 0 & $Q_{5}$ \\
$22: 43: 40.7$ & 90 & 0 & 45 & $Q_{5}$ \\
$22: 44: 12.9$ & 90 & 0 & 22.5 & $U_{5}$ \\
$22: 44: 50.8$ & 90 & 0 & 67.5 & $U_{5}$ \\
$22: 47: 45.5$ & 45 & 90 & 0 & $Q_{6}$ \\
$22: 48: 22.1$ & 45 & 90 & 45 & $Q_{6}$ \\
$22: 48: 56.3$ & 45 & 90 & 22.5 & $U_{6}$ \\
$22: 49: 34.3$ & 45 & 90 & 67.5 & $U_{6}$ \\
\hline
\end{tabular}


Table 5. A list of the compenents we measure with the different instrumental setups of Tab. 4. All components are listed in the form $X_{s k y} \rightarrow X_{U T 4} \rightarrow X_{N C}$, but we do not state the subscripts.

$$
\begin{array}{cllll}
Q_{5}= & +(I \rightarrow I \rightarrow Q) & +(I \rightarrow Q \rightarrow Q) & +(I \rightarrow U \rightarrow Q) & +(I \rightarrow V \rightarrow Q) \\
& +(U \rightarrow I \rightarrow Q) & +(U \rightarrow Q \rightarrow Q) & +(U \rightarrow U \rightarrow Q) & +(U \rightarrow V \rightarrow Q) \\
Q_{6}= & -(I \rightarrow I \rightarrow Q) & +(I \rightarrow Q \rightarrow Q) & +(I \rightarrow U \rightarrow Q) & -(I \rightarrow V \rightarrow Q) \\
& -(U \rightarrow I \rightarrow Q) & +(U \rightarrow Q \rightarrow Q) & +(U \rightarrow U \rightarrow Q) & -(U \rightarrow V \rightarrow Q) \\
Q_{7}= & +(I \rightarrow I \rightarrow Q) & +(I \rightarrow Q \rightarrow Q) & +(I \rightarrow U \rightarrow Q) & +(I \rightarrow V \rightarrow Q) \\
& -(U \rightarrow I \rightarrow Q) & -(U \rightarrow Q \rightarrow Q) & -(U \rightarrow U \rightarrow Q) & -(U \rightarrow V \rightarrow Q) \\
Q_{8}= & -(I \rightarrow I \rightarrow Q) & +(I \rightarrow Q \rightarrow Q) & +(I \rightarrow U \rightarrow Q) & -(I \rightarrow V \rightarrow Q) \\
& +(U \rightarrow I \rightarrow Q) & -(U \rightarrow Q \rightarrow Q) & -(U \rightarrow U \rightarrow Q) & +(U \rightarrow V \rightarrow Q) \\
& & & & \\
U_{5}= & +(I \rightarrow I \rightarrow U) & +(I \rightarrow Q \rightarrow U) & +(I \rightarrow U \rightarrow U) & +(I \rightarrow V \rightarrow U) \\
& +(U \rightarrow I \rightarrow U) & +(U \rightarrow Q \rightarrow U) & +(U \rightarrow U \rightarrow U) & +(U \rightarrow V \rightarrow U) \\
U_{6}= & -(I \rightarrow I \rightarrow U) & +(I \rightarrow Q \rightarrow U) & +(I \rightarrow U \rightarrow U) & -(I \rightarrow V \rightarrow U) \\
& -(U \rightarrow I \rightarrow U) & +(U \rightarrow Q \rightarrow U) & +(U \rightarrow U \rightarrow U) & -(U \rightarrow V \rightarrow U) \\
U_{7}= & +(I \rightarrow I \rightarrow U) & +(I \rightarrow Q \rightarrow U) & +(I \rightarrow U \rightarrow U) & +(I \rightarrow V \rightarrow U) \\
& -(U \rightarrow I \rightarrow U) & -(U \rightarrow Q \rightarrow U) & -(U \rightarrow U \rightarrow U) & -(U \rightarrow V \rightarrow U) \\
U_{8}= & -(I \rightarrow I \rightarrow U) & +(I \rightarrow Q \rightarrow U) & +(I \rightarrow U \rightarrow U) & -(I \rightarrow V \rightarrow U) \\
& +(U \rightarrow I \rightarrow U) & -(U \rightarrow Q \rightarrow U) & -(U \rightarrow U \rightarrow U) & +(U \rightarrow V \rightarrow U)
\end{array}
$$




\section{REFERENCES}

[1] Witzel, G., Eckart, A., Buchholz, R. M., Zamaninasab, M., Lenzen, R., Schödel, R., Araujo, C., Sabha, N., Bremer, M., Karas, V., Straubmeier, C., and Muzic, K., "The instrumental polarization of the Nasmyth focus polarimetric differential imager NAOS/CONICA (NACO) at the VLT. Implications for time-resolved polarimetric measurements of Sagittarius A*," Astronomy \& Astrophysics 525, 130 (Jan. 2011).

[2] Beuzit, J.-L., Feldt, M., Dohlen, K., Mouillet, D., Puget, P., Antichi, J., Baruffolo, A., Baudoz, P., Berton, A., and Boccaletti, A., "SPHERE: a'Planet Finder'Instrument for the VLT," The messenger 125, 29 (2006).

[3] Schmid, H. M., Downing, M., Roelfsema, R., Bazzon, A., Gisler, D., Pragt, J., Cumani, C., Salasnich, B., Pavlov, A., Baruffolo, A., Beuzit, J.-L., Costille, A., Deiries, S., Dohlen, K., Dominik, C., Elswijk, E., Feldt, M., Kasper, M., Mouillet, D., Thalmann, C., and Wildi, F., "Tests of the demodulating CCDs for the SPHERE / ZIMPOL imaging polarimeter," in [ Ground-based and Airborne Instrumentation for Astronomy $I V$. Proceedings of the SPIE], ETH Zürich (Switzerland) (Sept. 2012).

[4] Lenzen, R., Hartung, M., Brandner, W., Finger, G., Hubin, N. N., Lacombe, F., Lagrange, A.-M., Lehnert, M. D., Moorwood, A. F. M., and Mouillet, D., "NAOS-CONICA first on sky results in a variety of observing modes," in [Astronomical Telescopes and Instrumentation], Iye, M. and Moorwood, A. F. M., eds., 944-952, SPIE (Mar. 2003).

[5] Rousset, G., Lacombe, F., Puget, P., Hubin, N. N., Gendron, E., Fusco, T., Arsenault, R., Charton, J., Feautrier, P., Gigan, P., Kern, P. Y., Lagrange, A.-M., Madec, P.-Y., Mouillet, D., Rabaud, D., Rabou, P., Stadler, E., and Zins, G., "NAOS, the first AO system of the VLT: on-sky performance," in [Astronomical Telescopes and Instrumentation], Wizinowich, P. L. and Bonaccini, D., eds., 140-149, SPIE (Feb. 2003).

[6] Girard, J. H. V., Kasper, M., Quanz, S. P., Kenworthy, M. A., Rengaswamy, S., Schödel, R., Gallenne, A., Gillessen, S., Huerta, N., Kervella, P., Kornweibel, N., Lenzen, R., Mérand, A., Montagnier, G., O'Neal, J., and Zins, G., "Status and new operation modes of the versatile VLT/NaCo," in [Society of Photo-Optical Instrumentation Engineers (SPIE) Conference Series], Society of Photo-Optical Instrumentation Engineers (SPIE) Conference Series 7736 (July 2010).

[7] Canovas, H., Ménard, F., Hales, A., Jordan, A., Schreiber, M. R., Casassus, S., Gledhill, T. M., and Pinte, C., "Near-infrared imaging polarimetry of HD 142527," Astronomy \& Astrophysics 556, 123 (Aug. 2013).

[8] Garufi, A., Quanz, S. P., Avenhaus, H., Buenzli, E., Dominik, C., Meru, F., Meyer, M. R., Pinilla, P., Schmid, H. M., and Wolf, S., "Small vs. large dust grains in transitional disks: do different cavity sizes indicate a planet?," Astronomy 80 Astrophysics 560, A105 (Dec. 2013).

[9] Norris, B. R. M., Tuthill, P. G., Ireland, M. J., Lacour, S., Zijlstra, A. A., Lykou, F., Evans, T. M., Stewart, P., and Bedding, T. R., "A close halo of large transparent grains around extreme red giant stars," Nature 484, 220-222 (Apr. 2013).

[10] Quanz, S. P., Avenhaus, H., Buenzli, E., Garufi, A., Schmid, H. M., and Wolf, S., "Gaps in the HD 169142 Protoplanetary Disk Revealed by Polarimetric Imaging: Signs of Ongoing Planet Formation?," The Astrophysical Journal Letters 766, L2 (Mar. 2013).

[11] Avenhaus, H., Quanz, S. P., Schmid, H. M., Meyer, M. R., Garufi, A., Wolf, S., and Dominik, C., "Structures in the protoplanetary disk of HD142527 seen in polarized scattered light," The Astrophysical Journal 781, 87 (Feb. 2014).

[12] Harrington, D. M., Kuhn, J. R., and Hall, S., "Deriving Telescope Mueller Matrices Using Daytime Sky Polarization Observations," Publications of the Astronomical Society of the Pacific 123, 799-811 (July 2011).

[13] Tinbergen, J., [Astronomical Polarimetry] (Sept. 1996).

[14] Canovas, H., Rodenhuis, M., Jeffers, S. V., Min, M., and Keller, C. U., "Data-reduction techniques for high-contrast imaging polarimetry. Applications to ExPo," Astronomy \& Astrophysics 531, A102 (July 2011).

[15] van Harten, G., Snik, F., and Keller, C. U., "Polarization Properties of Real Aluminum Mirrors, I. Influence of the Aluminum Oxide Layer," Publications of the Astronomical Society of the Pacific 121, 377-383 (Apr. 2009).

[16] Witzel, G., Eckart, A., Lenzen, R., and Straubmeier, C., "On the Instrumental Polarisation of NAOSCONICA," The messenger 142, 5-7 (Dec. 2010). 